\title{
BMJ Open Guided self-determination intervention versus attention control for people with type 2 diabetes in outpatient clinics: a protocol for a randomised clinical trial
}

\author{
Anne Sophie Mathiesen (D) , ${ }^{1}$ Vibeke Zoffmann, ${ }^{2,3}$ Tine Bruhn Skytte, ${ }^{2}$ \\ Janus C Jakobsen, ${ }^{4,5}$ Christian Gluud, ${ }^{5,6}$ Jane Lindschou, ${ }^{5}$ Bodil Rasmussen, ${ }^{7,8}$ \\ Emilie Marqvorsen, ${ }^{2}$ Thordis Thomsen, ${ }^{9,10}$ Mette Rothmann ${ }^{4,7}$
}

To cite: Mathiesen AS, Zoffmann V, Skytte TB, et al. Guided self-determination intervention versus attention control for people with type 2 diabetes in outpatient clinics: a protocol for a randomised clinical trial. BMJ Open 2021;11:e047037. doi:10.1136/ bmjopen-2020-047037

- Prepublication history for this paper is available online. To view these files, please visit the journal online (http://dx.doi. org/10.1136/bmjopen-2020047037).

Received 18 November 2020 Accepted 22 November 2021

Check for updates

(C) Author(s) (or their employer(s)) 2021. Re-use permitted under CC BY-NC. No commercial re-use. See rights and permissions. Published by BMJ.

For numbered affiliations see end of article.

Correspondence to Dr Anne Sophie Mathiesen; asmathiesen@gmail.com

\section{ABSTRACT}

Introduction In the management of type 2 diabetes, autonomy-supporting interventions may be a prerequisite to achieving more long-term improvement. Preliminary evidence has shown that the guided self-determination (GSD) method might have an effect on haemoglobin A1c and diabetes distress in people with type 1 diabetes. Previous trials were at risk of uncertainty. Thus, the objective is to investigate the benefits and harms of a GSD intervention versus an attention control group intervention in adults with type 2 diabetes.

Methods and analysis This trial protocol is guided by the The Standard Protocol Items: Recommendations for International Trials Statement. We describe the protocol for a pragmatic randomised, dual-centre, parallel-group, superiority clinical trial testing a GSD intervention versus an attention control for people with type 2 diabetes in outpatient clinics. The participants $(n=224)$ will be recruited from two diverse regions of Denmark. The experimental stepped-care intervention will consist of three to five GSD sessions lasting up to 1 hour with a trained GSD facilitator. The sessions will be conducted face to face, by video conference or over the telephone. The attention controls will receive three to five sessions lasting up to an hour with a communication-trained healthcare professional provided face to-face, by video conference, or over the telephone. Participants will be included if they have type 2 diabetes, $>18$ years old, are not pregnant. Participants will be assessed before randomisation, at 5-month, and 12-month follow-up, the latter being the primary. The primary outcome is diabetes distress. Secondary outcomes are quality of life, depressive symptoms and non-serious adverse events. Exploratory outcomes are haemoglobin A1c, motivation and serious adverse events. Data will be collected using REDCap and analysed using Stata V.16.

Ethics and dissemination The trial will be conducted in compliance with the protocol, the Helsinki Declaration in its latest form, International Harmonisation of Good Clinical Practice guidelines and the applicable regulatory requirement(s). The trial has been approved by the Danish Data Protection Agency (P-2020-864). The Ethics Committee of the Capital Region of Denmark reviewed the trial protocol, but exempted the trial protocol from full review $(\mathrm{H}-20003638)$. The results of the trial will be

\section{Strengths and limitations of this study}

- We conducted a systematic review before initiating the trial to justify the rationale for the trial.

- To decrease the risk of a 'Hawthorne effect' and other biases an attention control group was included.

- The trial is designed as a pragmatic trial with few inclusion and exclusion criteria, which may increase the generalisability of the results to clinical practice.

- We conducted power calculations for all secondary outcomes.

- The trial is only a two-centre trial, planning to include a limited population of about 224 participants.

presented at the outpatient clinics treating people with type 2 diabetes, at national and international conferences as well as to associations for people with diabetes and their relatives.

Trial registration number ClinicalTrials.gov identifier: NCT04601311.

\section{INTRODUCTION}

Diabetes affects 425 million people worldwide, and of these, type 2 diabetes accounts for $90 \%{ }^{1}$ The prevalence and incidence of type 2 diabetes in particular is rapidly rising. ${ }^{1}$ Type 2 diabetes is caused by a genetic disposition in combination with a sedentary lifestyle and overweight. ${ }^{2}$ With age being the largest risk factor, the number of people living with type 2 diabetes and various combinations of comorbidities is also increasing. ${ }^{13}$

Complications of type 2 diabetes include macrovascular complications such as ischaemic stroke or coronary heart disease. $^{2} \quad 4$ Microvascular complications comprise retinopathy, neuropathy and nephropathy. ${ }^{4} \mathrm{Up}$ to one-third of people with type 2 diabetes have developed one or more complications of type 2 diabetes at the time of diagnosis. ${ }^{5}$ 
Type 2 diabetes management aims to prevent or reduce complications of diabetes. An appropriate management plan should consider the person's age, cognitive abilities, literacy, social and financial situation, cultural factors, diabetes complications and comorbidities, and health priorities including preferences of care. ${ }^{6}$ Performing recommended self-care behaviours is challenging, particularly for populations with other chronic diseases. ${ }^{7}$ People with type 2 diabetes stratified for outpatient clinics have a high degree of comorbidity in addition to adverse psychosocial outcomes such as diabetes distress and depressive symptoms. ${ }^{8}{ }^{9}$ Inadequate diabetes self-management is associated with worse psychosocial outcomes. ${ }^{10}$

Diabetes self-management depends on the individual's motivation and autonomy. Autonomy will underpin the planned intervention because satisfactory diabetes selfmanagement is easier to accomplish and maintain if the individual's motivation is autonomous-meaning that the individual strives for goals they genuinely believe in. ${ }^{11}$

Thus, autonomy-supporting interventions and intrinsic motivation may be crucial factors in achieving real-life patient engagement and more long-term improvement through shared decision-making and collaborative goal setting. ${ }^{12}{ }^{13}$ A method designed to promote autonomy and intrinsic motivation in people with diabetes is the guided self-determination (GSD) method-an empowermentbased method recognised as a life-skills approach ${ }^{14}$ clinically applicable in person within diabetes-provider relationships. ${ }^{15-19}$ In the GSD approach, the person with diabetes has the primary role of preparing for consultations at home, by filling in reflection sheets, which enables the person to clarify and prioritise what is important to change, thus becoming able to express their thoughts in communication with the healthcare professionals. Subsequently, they may improve clinical outcomes ${ }^{19-21}$ through the following pathways ${ }^{15-17}$ : increased perceived autonomy support from the healthcare professionals, increased perceived competence in managing diabetes, decreased diabetes-related distress and ultimately improved glycaemic control. ${ }^{18} 19$

We previously conducted a systematic review and meta-analysis to investigate the evidence of psychosocial interventions on diabetes distress, HbAlc, depression and health-related quality of life in people with type 2 diabetes. ${ }^{22}$ We found small effects of psychosocial interventions on diabetes distress and depressive symptoms, but the risk of bias and heterogeneity of included trials were high and the certainty of the evidence was very low to moderate. ${ }^{22}$ To investigate autonomy-supportive interventions specifically, we searched the Cochrane Database of Systematic Reviews and PubMed using the search terms: type 2 diabetes, theory-based interventions, GSD, self-determination theory, illness integration theory and person-centred approaches in different combinations. From the searches, we identified only five randomised clinical trials investigating the effect of GSD in people with type 1 diabetes. ${ }^{19212324}$ The trial results showed statistically significant improvements in glycaemic control, ${ }^{19}$ diabetes distress ${ }^{21}$ and diabetes competences. ${ }^{1921}$ We identified no systematic reviews assessing the effects of GSD. Thus, we also conducted a systematic review investigating the effect of GSD or self-determination theory-based interventions versus standard care in people with diabetes..$^{25}$ However, all identified randomised clinical trials were at high risk of bias and the certainty of the evidence was low, which might entail that the previous trials overestimated the beneficial effects of GSD. ${ }^{26}$ Furthermore, none of the trials described adverse events.

We here describe our new randomised clinical trial going to assess GSD versus attention control for people with type 2 diabetes. Our choice of the attention control group is based on the most recent international recommendations on type 2 diabetes management. ${ }^{3}$ Additionally, we provide up to five contacts with a research assistant trained in supportive communication techniques to the participants in the control group. The inclusion of an attention control group is meant to alleviate expectation bias and reduce attrition ${ }^{27}$ as well as the effects of the Hawthorne effect. ${ }^{28}$

\section{OBJECTIVES}

The primary objective is to assess if GSD intervention versus attention control in people with type 2 diabetes:

- Reduces diabetes distress with at least six points on the Problem Areas in Diabetes (PAID) Scale. ${ }^{29}$

The secondary objectives are to assess if GSD intervention versus attention control for people with type 2 diabetes:

- Reduces depressive symptoms and anxiety with at least three points on the Hospital Anxiety and Depression Scale (HADS). ${ }^{30} 31$

- Improves physical and mental quality of life with at least six points on the SF-36 Scale. ${ }^{32} 33$

- Reduces adverse events with at least five points on the Negative Effects Questionnaire 20-items (NEQ20). ${ }^{34} 35^{\circ}$

Furthermore, the exploratory objectives are to assess if GSD intervention versus attention control in adults with type 2 diabetes:

- Improves serum HbA1c.

- Motivation measured by the Treatment Self-Regulation Questionnaire (TSRQ).

- Reduces the proportion of participants with one or more serious adverse events in the intervention period according to the International Harmonisation of Good Clinical Practice (ICH-GCP) definition. ${ }^{36}$

\section{METHODS AND DESIGN \\ Design}

The trial is designed as a pragmatic, investigator-initiated, dual-centre, randomised, parallel-group, assessorblinded, superiority clinical trial of persons with type 2 diabetes. Participants $(n=224)$ will be recruited from the Department of Endocrinology, the University Hospital of Copenhagen-Rigshospitalet, The Capital Region of 


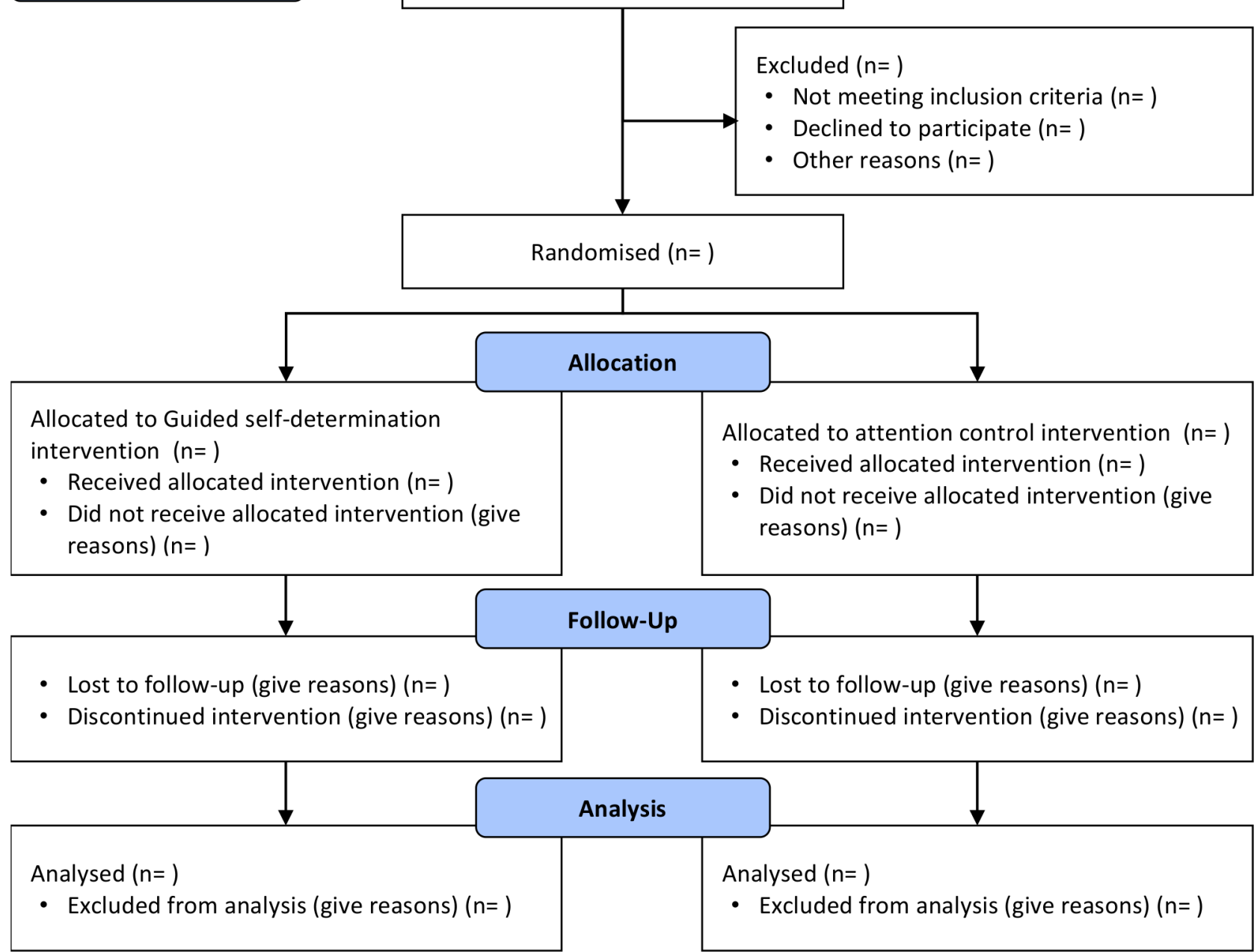

Figure 1 Flowchart of the trial.

Denmark and Steno Diabetes Center Odense, Odense University Hospital, The Southern Region of Denmark. We adhere to the The Standard Protocol Items: Recommendations for International Trials Statement regarding conduct $^{37}$ and the Consolidated Standards of Reporting Trials Statement extension for non-pharmacologic treatment interventions regarding reporting of this trial. ${ }^{38} 39$ Initially, the planned start date was 15 August 2020, but the start was delayed due Denmark's first wave of COVID19. Consequently, the first participant was randomised on 11 November 2020. The second wave of COVID-19 (from December 2020 to March 2021) meant further delay for the trial. The last date of follow-up for the last participant is expected to be in June 2023. Data analysis and publication of results are expected to be carried out from July to December 2023. Flowchart of the trial figure 1: the flowchart $(n=224)$ will be filled in at the end of the trial. As part of this trial, we also plan for a nested qualitative study to elaborate on the mechanism of action in people with type 2 diabetes as well as the online versus face-to-face preferences and barriers.

\section{Patient and public involvement}

The development of the GSD method is based on a comprehensive involvement of people with diabetes. ${ }^{15-17}$ The results of the trial will be presented to the participants and their relatives and associations for people with diabetes in Denmark and abroad, such as the Danish Association of Diabetes and Diabetes UK.

\section{Trial sites and personnel}

Both trial sites are specialised in type 2 diabetes with high complexity (ie, high degree of comorbidity and/or complications); thus, participants recruited for this trial are likely to have type 2 diabetes and one or more complications and/or comorbidities.

Registered nurses will be recruited for training and certification in the GSD method. The nurses undergoing training will be certified in the GSD method after a 32-hour structured and supervised training course. Subsequently, the nurses will be required to document their ability to use the reflection sheets and communication skills in two supervised courses with people with diabetes. After the courses, they will be required to fill in a self-assessment 
form, which is discussed with Vibeke Zoffmann to finalise their individual certification as GSD facilitators. During the training period, models from the three grounded theories will be used as fidelity assessment tools.

\section{Selection of participants}

All people with diabetes referred to, or followed at the participating clinical trial sites (Department of Endocrinology, University Hospital of Copenhagen-Rigshospitalet and Steno Diabetes Center Odense, Odense University Hospital) are considered for participation and will be eligible, if they comply with the inclusion and exclusion criteria indicated below.

\section{Inclusion criteria}

- Being 18 years of age or older.

- Having been diagnosed with type 2 diabetes for three or less months according to the International Classification System of Diseases (ICD-11.2-11.9)

- Having signed informed consent.

\section{Exclusion criteria}

- Pregnancy; women who are premenopausal will be asked if they are pregnant or are planning pregnancy prior to inclusion.

- Prior participation in GSD course(s) for the past two years.

- Lack of signed informed consent.

\section{Screening and informed consent}

Potentially eligible participants will be approached and screened for eligibility by clinical staff (nurses/dieticians/physicians) at the outpatient clinics. Study nurses at each site will screen record to identify potentially eligible participants.

If eligible, detailed written patient information will be provided in person or by email and supplemented by verbal information. This information is only provided by the GSD certified nurses or the investigators. The clinical personnel will provide the information on the trial in an undisturbed room at the clinic. It will be stressed that the eligible person with diabetes will have the opportunity of bringing a third party (eg, a relative or friend). Eligible persons will be given at least 2 days to reflect before deciding to participate and will sign informed consent prior to inclusion and collection of any data. Due to our pragmatic design with few inclusion and exclusion criteria, no prior testing of participants' eligibility will be necessary.

\section{Baseline assessments}

We will collect the following data: sex, age, marital status, duration of diagnosis, employment, educational level, diabetes treatment, medications, number of comorbidities (complications of diabetes and other comorbidities). Data on diabetes-related comorbidities are retinopathy, nephropathy and neuropathy. Other comorbidities are defined as psychiatric comorbidities (depression, anxiety, schizophrenia, bipolar disorder) and medical comorbidities (cardiovascular disease, cancer, musculoskeletal disease, liver disease, apoplexy, abuse diagnoses). Baseline information will be collected from participants' records.

\section{Randomisation}

Participants will be centrally randomised at a 1:1 ratio using a web-based system developed by The Copenhagen Trial Unit. The allocation will be computer-generated through permuted blocks of varying sizes and concealed to the investigators. The certified GSD nurses will enrol the participants and assign the interventions. The randomisation will be stratified according to (1) centre and (2) sex, the latter due to a better effect on HbAlc and diabetes distress in women than men. ${ }^{21}$

\section{Experimental intervention}

The GSD is a theory-based and evidence-based problemsolving method to overcome barriers to collaborative care. It is a life-skills approach ${ }^{14}$ that strives at promoting autonomous motivation, empowerment and self-determination. Its focus areas are life-illness integration, ${ }^{16}$ relational potential for change and shared decision-making. ${ }^{15} 17$ These concepts are integrated into worksheets which are essential to the practical application of the method. The GSD method entails advanced professional communication skills such as active listening, mirroring and valuesclarifying responses to facilitate autonomous reflection in the people with diabetes focusing on diabetes management issues perceived as challenging. ${ }^{15}$

Experienced diabetes nurses certified in the GSD method provide the experimental intervention as a stepped-care intervention to each participant individually. Before randomisation, both the intervention and the control group will be supported in formulating one personal value-clarifying goal: 'One thing I want to achieve in my life with diabetes within a year is [....]'.

Within the first 4 months after randomisation, the participant will receive two to five need-based sessions with the GSD facilitator that will be conducted face to face, by video or over the telephone. The number of conversations will be decided by the participant-facilitator dyad during the second session based on the participant's perceived need. Facilitators will be unaware of the participant's level of diabetes distress, depression and anxiety scores. The stepped-care intervention will be provided as a digital version, an analogue GSD (in paper) or a mixed version as preferred by the participant.

The GSD intervention will require participants to complete 13 reflection sheets in a predefined order as preparation for five individual sessions scheduled every second week (figure 2). The sheets will be handwritten or digitally written on pages hosted at the Danish national health portal, Sundhed.dk. Additionally, a set of five sheets on motivation for evidence-based glucose control will be used in an analogue format. It will be possible to share and discuss the digital pages completed on the Sundhed.dk's platform face to face, over phone, or by 


\section{Overview of sessions}

Information, written consent, $\mathrm{HbA} 1 \mathrm{c}$ and baseline questionnaires.

Personal goal stated by answering the unfinished sentence:

One thing I want to achieve in my life with diabetes within a year is...

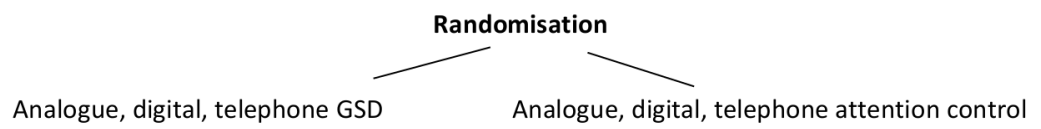

Session 0 face-to-face

Analogue, digital, telephone attention control

Intervention group

Invitation to collaboration is reviewed

Choice of digital, analogue or combined GSD

Provision of reflection sheets/SDK pages for meeting 1

Meeting 1 and 2 are scheduled

Session 0 face-to-face

Session 1 ( 2 weeks after)

Your body - what you feel, what you see

Attention control group

Introduction to phone

contact person

Personal goal registered in

Redcap

Symptoms in everyday life with diabetes

Visit 1 ( 2 weeks after)

Important events and periods

What is currently challenging or difficult?

Unfinished sentences (1-2 / 2)

Make a drawing or write some words

Session 2 (4 weeks after)

Session 2 (4 weeks after)

Relatives or companion's unfinished sentences (when needed)

Follow-up on personal goal

Your week

Your everyday life and plans to change your lifestyle

For and against

Room for diabetes in my life

\section{TIMEOUT}

Patient's \& nurse's lists are updated

Agreement on problem/challenge to focus on '

Session 3 ( 6 weeks after)

Dynamic judgment building

Your observations

Your thoughts

Your goals and intentions

Your actions

Problem solving for now and in the futur

Session 4 ( 8 weeks after)

\section{Short version:}

Meetings 1-2-4-5

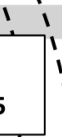

Session 3 (6 weeks after)

Follow-up on personal goal

Session 4 (8 weeks after)

Follow-up on personal goal

Blood glucose measurements and my reasons for measuring

My ideal blood sugar values

What can be gained by lowering $\mathrm{HbA} 1 \mathrm{c}$ ?

What can I gain from my ideal HbA1c goal?

f́,

Session 5 (10 weeks after)

Your outcome in one sentence

Follow-up on personal goal

Changes you are still working on

Topics you want to talk to others about

Schedule next appointment at the outpatient clinic

Schedule next appointment at the outpatient

with relevant $\mathrm{HCPs}$ and collaborators

Figure 2 Overview of sessions. GSD, guided self-determination. HCP, healthcare professional

a secure video function called 'Hi Doctor'. Participants opting for a digital session will be able to access Sundhed. $\mathrm{dk}$ through a link provided by the certified GSD facilitator and complete the reflection sheets as preparation for the session.
Each session will have a standard duration of up to 1 hour. Participants will have the opportunity to bring a relative or a friend to the second section. The relative or friend will also have completed a reflection sheet as preparation. An overview of visits and the reflection sheets are 
presented in figure 2. Reasons for a short version 1-2-4-5 and a short version 1-2-5 are low complexity and consequently reduced needs, respectively. The total number of reflection sheets filled in by the participants will be recorded in REDCap to document adherence to the offered intervention.

\section{Control intervention}

The participants in the control group will receive an attention control intervention that will include the following components: (1) before randomisation, the attention control group is supported in formulating one personal value-clarifying goal: 'One thing I want to achieve in my life with diabetes within a year is [...]'; (2) the participant's personal goal will be registered by a communication-trained healthcare professional (the communication applied by the communication-trained healthcare professional will be advanced as she will have communication skills as a certified counsellor); (3) the communication-trained healthcare professional will follow-up the goal by contacting the participants in the control group 2 weeks and 1 month after randomisation to hear how they are doing with the goal, and if they wish to continue with three more sessions provided face-toface, by video, or over telephone. The decision on three more session will be needs-defined. These needs-defined sessions will be scheduled concurrently with the sessions in the experimental group, namely 6 weeks, 8 weeks and 10 weeks after randomisation; and (4) all sessions will last up to 1 hour. The relatively short intervention has been qualitatively evaluated ${ }^{40}$ and aims at being applicable in clinical practice in a population with a high degree of comorbidity. An overview of contacts in the attention control group is presented in figure 2 .

\section{Cointerventions}

Both groups will receive standard treatments, including a $20 \mathrm{~min}$ visit to their primary care physician every 5-6 months. Both groups will receive follow-up questionnaires electronically at 5-month and 12-month follow-up. Facilitators and investigators will be alerted if participants do not respond to the questionnaires and the two subsequent reminders, after which the participant will be contacted by phone.

\section{Concomitant interventions}

Participants will continue with their usual antidiabetic medication regime during the trial and follow-up periods and they will be asked about their current treatment at each session also through follow-up contacts. Any initiation or discontinuation of medication (including antidiabetics) will be recorded.

\section{Outcomes}

For an overview of all outcomes and their corresponding time of assessment, see table 1 .

\section{Primary outcome}

- Diabetes distress at 12-month follow-up assessed by the validated 20-item scale of diabetes-related distress burden, PAID. ${ }^{29}{ }^{41}$ The sum of items ranging from 0 (not a problem) to 4 (a serious problem) will be transformed into a 0 to 100 sum score by multiplying by 1.25. A score $\geq 30$ will indicate high diabetes distress. ${ }^{29}$ PAID will be measured at baseline and at 5-month and

Table 1 Outcomes and timepoints

\begin{tabular}{|c|c|c|c|c|}
\hline & Enrolment & $\begin{array}{l}\text { Allocation } \\
\text { (baseline) }\end{array}$ & Follow-up & \\
\hline Timepoint & $-\mathrm{t} 1$ & 0 & 5 months & 12 months \\
\hline \multicolumn{5}{|l|}{ Attention control } \\
\hline $\begin{array}{l}\text { Assessments: socio-demographic factors (at baseline only), } \\
\text { diabetes-related comorbidities, psychiatric comorbidities } \\
\text { and medical comorbidities }\end{array}$ & $x$ & $x$ & & \\
\hline Problem Areas in Diabetes & & $\mathrm{X}$ & $x$ & $X$ \\
\hline Serum HbA1c & & $x$ & & $x$ \\
\hline Severe hypoglycaemia & & & & $x$ \\
\hline $\begin{array}{l}\text { Diabetes medication at } 5 \text {-month and } 12 \text {-month follow-up } \\
\text { change in diabetes medication }\end{array}$ & & $x$ & $x$ & $x$ \\
\hline
\end{tabular}


12-month follow-up, with 12 months as the primary follow-up timepoint.

\section{Secondary outcomes}

- Depressive symptoms assessed by the HADS ${ }^{30} 31$ with 14 questions ranging from 0 to 3 divided into two subscales for depression and anxiety. In this trial, we will apply the cut-off points for the total scale, as recommended in populations with minor comorbid psychiatric disorders. ${ }^{42}$ Scores $>13$ indicate moderate depression and $>19$ indicate a major depressive disorder. HADS will be measured at 12-month follow-up. ${ }^{42} 43$

- Generic quality of life assessed by SF-36, V.1.0, consisting of 36-items divided into a physical and mental domain with eight subscales: general health, physical functioning, social functioning, mental health, physical role, emotional role, bodily pain and vitality. We will assess the physical and mental scale at 12-month follow-up. ${ }^{32} 33$

- Adverse events ${ }^{36}$ in the intervention period assessed by the NEQ-20 consisting of 20-items on a 5-point Likert scale assessed at 12 month follow-up. ${ }^{34} 35$

\section{Exploratory outcomes}

- Serum HbAlc concentration will be assessed from the participants' records at 12-months \pm 2 -week follow-up.

- Type of motivation (autonomous/external), controlled (external) or resigned (amotivated) regarding diabetes self-care practices will be assessed by the TSRQ. ${ }^{44}$ The TSRQ consists of 21 items divided into three subscales: (1) autonomous (eight items); (2) controlled behaviour (nine items); and (3) amotivated (four items). TSRQ will be measured at 12-month follow-up.

- Proportion of participants with one or more serious adverse events in the intervention period, defined according to the International Harmonisation of Good Clinical Practice definition, as any untoward medical occurrence that resulted in death, was life-threatening, required hospitalisation or the prolonging of existing hospitalisation, and resulted in persistent or significant disability or jeopardised the patient. ${ }^{36}$

\section{Blinding}

Participants and treatment providers will not be blinded to the allocated trial intervention. The treatment providers will not be involved in the analyses. All other medical personnel will be blinded to the notes in the participant's electronic records. Outcome assessors and external statisticians at The Copenhagen Trial Unit will be blinded to the participants' randomisation status. The statistical analyses will be conducted with the intervention groups coded as X and Y. After conducting the statistical analyses under code, the steering committee will write two abstracts while the blinding is intact-one assuming that the experimental intervention group is $\mathrm{X}$ and the control intervention group is $\mathrm{Y}$, and one assuming the opposite. After this, the code will be broken. ${ }^{45} 46$

\section{Participants' discontinuation and withdrawal}

Participants will be able to withdraw consent from participation in the trial at any time without reason and consequences for future treatment at the clinics. One of the investigators will contact the participant and ask which aspects of the trial they wish to withdraw from: (1) the trial intervention or control group; (2) the follow-up assessments; and/or (3) use of already collected data. Only if a participant wishes to fully withdraw from the abovementioned points, the data will not be used in the analyses. In all of the abovementioned cases, the trial investigators will encourage them to continue with the follow-up assessments.

Discontinuation of a participant at the choice of the investigators will happen if:

1. The participant gets pregnant during the intervention period, or

2. The participant experiences intolerable adverse reactions.

In all of the abovementioned cases, the trial investigators will encourage them to continue with the follow-up assessments. A Data Monitoring Commiittee is not required in non-pharmacological diabetes self-management interventions, ${ }^{36}$ but the trial management committee will monitor recruitment, treatment and attrition rates and any concerns related to the trial.

\section{Data management}

Data collection will be conducted by the certified faciliators using tablets and electronic case report form developed in the data collection management system REDCap. All self-reported questionnaires will be collected from REDCap. An overview of outcomes measures and data collection timepoints can be found in table 1 .

\section{Sample size}

The sample size was determined by a predicted difference in the primary outcome measure, PAID, between the experimental and control group. We could not identify any previous studies quantising the minimal important difference of PAID. Informed by the estimated mean and SD from the trials included in our systematic review ${ }^{25}$ (results not yet published), we pragmatically chose six PAID points as the minimal important difference. It must be noted that the minimal important difference should preferably be based on low risk of bias trials, as high risk of bias trials may overestimate the effect. ${ }^{26}$ Unfortunately, it was only possible to identify trials at high risk of bias in our systematic review. ${ }^{25}$ In another review conducted by our research group, ${ }^{22}$ we did, however, identify trials at low risk of bias investigating the effect of psychosocial interventions (not GSD). We found that estimates from these trials at low risk of bias ${ }^{47}$ roughly corresponded to the minimal important difference and SDs chosen for our sample size calculation. Consistent with these trials that 
used PAID as an outcome measure, we expect a SD of 16. With a power of $80 \%$ (a beta at 20\%) and an $\alpha$ at $5 \%$, two-tailed, a sample size of 112 participants is needed in each intervention group corresponding to a total of 224 participants included in the trial.

\section{Power calculation for the secondary outcomes}

Based on previous trials, ${ }^{32} 33$ an estimated minimal important difference of six points \pm SD of 15 on the SF-36 scale, $\alpha$ at $5 \%$, and 112 participants in each intervention group, we achieved a power of $84.9 \%$.

Based on previous trials, ${ }^{40} 48$ an estimated minimal important difference of three points \pm SD of six on the HADS scale, $\alpha$ at $5 \%$, and 112 participants in each intervention group, we achieve a power of $96.3 \%$.

No minimal clinically important difference based on previous trials has been reported when applying the NEQ-20. ${ }^{34}$ Thus, we pragmatically expect a difference of five points \pm SD of 13 on the NEQ-20, $\alpha$ at $5 \%$ and 112 participants in each group. Hence, we achieved a power of $82.1 \%$.

\section{Statistical methods}

All continuous outcomes will be assessed by linear regression and the dichotomous outcomes will be assessed by logistic regression. The primay outcome, PAID will be measured at baseline and at 5-month and 12-month follow-up, with 12 months as the primary follow-up timepoint. All secondary and exploratory outcomes will be assessed at baseline and 12-month follow-up. The analyses will be performed on the intention-to-treat and per-protocol basis, respectively, and will be adjusted for the stratification variables: site and sex. We will conduct subgroup analyses on educational level, sex and number of comorbidities.

The five-step procedure developed by Jakobsen et at ${ }^{49}$ will be applied to assess if the threshold for clinical and statistical significance is crossed. Missing data will be handled according to the recommendations by Jakobsen et $a l^{50}$ All analyses will be conducted blinded with the experimental and the control group concealed as $\mathrm{X}$ and Y. A detailed statistical analysis plan will be published prior to the analysis of trial data.

\section{Ethics and dissemination}

The trial will be conducted in compliance with the protocol, the Helsinki Declaration in its latest form, ${ }^{51}$ ICH-GCP guidelines, ${ }^{36}$ and the applicable regulatory requirement(s). The trial has been approved by the Danish Data Protection Agency (P-2020-864). The Ethics Committee of the Capital Region of Denmark reviewed the trial protocol on two occasions, but exempted the trial protocol from full review (H-20003638) dated 16 January and 18 August 2020.

\section{Dissemination policy}

The results of the trial will be presented at the outpatient clinics treating people with type 2 diabetes, at national and international conferences as well as to associations for people with diabetes and their relatives. Negative, positive or neutral results will be published in international peerreviewed journals. Following international guidelines, we will publish the anonymised individual participant data transparently together with our publication. ${ }^{52} 53$

\section{Perspectives}

The trial aims to provide evidence for or against an effect of GSD compared with attention controls in people with type 2 diabetes in an outpatient clinic setting. The GSD method has not yet been tested in this population and may help identify subgroups of people with diabetes for whom GSD may be more or less effective and the most efficient way of delivering it.

This information may potentially increase results' costeffectiveness and external validity. Likewise, their generalisability might be increased by the trial's pragmatic design including few exclusion criteria.

Furthermore, before planning this protocol, we conducted a systematic review using the Cochrane methodology to synthesise the evidence for the beneficial and harmful effects of GSD or self-determination theory interventions..$^{25}$ The systematic review considered both risks of random errors and systematic errors to identify critical gaps in existing trials investigating the GSD method. A potential limitation might be that a high level of literacy may be required to achieve the optimal benefit of the reflection sheets. ${ }^{54}$

\section{Trial status}

This is the first version of the trial protocol dated 9 October 2020. The first participant is scheduled for inclusion in November 2020.

\section{Author affiliations}

${ }^{1}$ Department of Endocrinology, Center for Cancer and Organ Diseases, Rigshospitalet, Copenhagen, Denmark

${ }^{2}$ The Research Unit Women's and Children's Health, The Julie Marie Center, Rigshospitalet, Copenhagen, Denmark

${ }^{3}$ Department of Public Health, University of Copenhagen, Sector of Public Health Service Research, Copenhagen, Denmark

${ }^{4}$ Department of Clinical Research, University of Southern Denmark, Odense, Denmark

${ }^{5}$ The Copenhagen Trial Unit, Centre for Clinical Intervention Research, Rigshospitalet, Copenhagen, Denmark

${ }^{6}$ Department of Regional Clinical Research, Faculty of Health Sciences, University of Southern Denmark, Odense, Denmark

${ }^{7}$ Steno Diabetes Center Odense, Odense University Hospital, Odense, Denmark

${ }^{8}$ Western Health Partnership, School of Nursing and Midwifery, Faculty of Health, Deakin University, Melbourne, Victoria, Australia

${ }^{9}$ Department of Anaesthesiology, Herlev University Hospital, Herlev, Denmark ${ }^{10}$ Department of Clinical Medicine, University of Copenhagen, Copenhagen, Denmark

\section{Twitter Tine Bruhn Skytte @tineskytte}

Acknowledgements We thank the Novo Nordisk Foundation, Steno Collaborative Grant, (grant number: NNF100C0057720) for an unconditional grant to support the trial. In the design of this trial protocol, we used The Copenhagen Trial Unit's standard operation procedure. We acknowledge the RAND collaboration for permission to use the V.1.0 of the SF-36.

Contributors Author contributions as stated in the manuscript: ASM, MR, VZ, $\mathrm{JL}, \mathrm{CG}, \mathrm{JCJ}$ and $\mathrm{TT}$ conceived the trial and led the development of all procedures 
including intervention design. ASM wrote up the protocol with regular supervision from MR, VZ, TT, JL, CG, JCJ and JL. TBS assisted in setting up procedures and data collection at the recruiting sites. BR, TBS and EM read and commented the final manuscript before it was submitted for publication. All authors read and approved the final manuscript.

Funding The study is funded by the Novo Nordisk Foundation, Steno Collaborative grant (grant number: NNF100C0057720).

Disclaimer The Novo Nordisk Foundation has not been involved in the design and will not be involved in the collection of data, analyses, interpretation of data, or in writing up the manuscript.

Competing interests The second author (VZ) has developed the GSD method, which may introduce author bias.

Patient and public involvement Patients and/or the public were involved in the design, or conduct, or reporting, or dissemination plans of this research. Refer to the Methods and design section for further details.

Patient consent for publication Not applicable.

Provenance and peer review Not commissioned; externally peer reviewed.

Open access This is an open access article distributed in accordance with the Creative Commons Attribution Non Commercial (CC BY-NC 4.0) license, which permits others to distribute, remix, adapt, build upon this work non-commercially, and license their derivative works on different terms, provided the original work is properly cited, appropriate credit is given, any changes made indicated, and the use is non-commercial. See: http://creativecommons.org/licenses/by-nc/4.0/.

\section{ORCID iD}

Anne Sophie Mathiesen http://orcid.org/0000-0002-1354-1012

\section{REFERENCES}

1 Karuranga S, Fernandes JR, Huang Y, et al. IDF diabetes atlas, 2017

2 Chatterjee S, Khunti K, Davies MJ. Type 2 diabetes. Lancet 2017;389:2239-51.

3 American Diabetes Association. 6. Glycemic targets: standards of medical care in diabetes-2018, 2018.

4 Gregg EW, Sattar N, Ali MK. The changing face of diabetes complications. Lancet Diabetes Endocrinol 2016;4:537-47.

5 Gedebjerg A, Almdal TP, Berencsi K, et al. Prevalence of micro- and macrovascular diabetes complications at time of type 2 diabetes diagnosis and associated clinical characteristics: a cross-sectional baseline study of 6958 patients in the Danish DD2 cohort. J Diabetes Complications 2018;32:34-40.

6 Davies MJ, D'Alessio DA, Fradkin J, et al. Management of hyperglycaemia in type 2 diabetes, 2018. A consensus report by the American diabetes association (ADA) and the European association for the study of diabetes (EASD). Diabetologia 2018;61:2461-98.

7 Rosbach M, Andersen JS. Patient-experienced burden of treatment in patients with multimorbidity - A systematic review of qualitative data. PLoS One 2017;12:e0179916.

8 Fisher L, Mullan JT, Skaff MM, et al. Predicting diabetes distress in patients with type 2 diabetes: a longitudinal study. Diabet Med 2009;26:622-7.

9 Stoop CH, Nefs G, Pop VJ, et al. Diabetes-specific emotional distress in people with type 2 diabetes: a comparison between primary and secondary care. Diabet Med 2014;31:1252-9.

10 Fisher L, Glasgow RE, Strycker LA. The relationship between diabetes distress and clinical depression with glycemic contro among patients with type 2 diabetes. Diabetes Care 2010;33:1034-6.

11 Sheldon KM, Elliot AJ. Goal striving, need satisfaction, and longitudinal well-being: the self-concordance model. J Pers Soc Psychol 1999;76:482-97.

12 Ntoumanis N, Ng JYY, Prestwich A, et al. A meta-analysis of selfdetermination theory-informed intervention studies in the health domain: effects on motivation, health behavior, physical, and psychological health. Health Psychol Rev 2021;15:214-44.

13 Gillison FB, Rouse P, Standage M, et al. A meta-analysis of techniques to promote motivation for health behaviour change from a self-determination theory perspective. Health Psychol Rev 2019;13:110-30.

14 Nutbeam DON. Health promotion glossary. Health Promot Int 1986;1:113-27.

15 Zoffmann V, Harder I, Kirkevold M. A person-centered communication and reflection model: sharing decision-making in chronic care. Qual Health Res 2008;18:670-85.
16 Zoffmann V, Kirkevold M. Life versus disease in difficult diabetes care: conflicting perspectives disempower patients and professionals in problem solving. Qual Health Res 2005;15:750-65.

17 Zoffmann V, Kirkevold M. Relationships and their potential for change developed in difficult type 1 diabetes. Qual Health Res 2007; 17:625-38.

18 Zoffmann V, Kirkevold M. Realizing empowerment in difficult diabetes care: a guided self-determination intervention. Qual Health Res 2012;22:103-18.

19 Zoffmann V, Lauritzen T. Guided self-determination improves life skills with type 1 diabetes and $\mathrm{A} 1 \mathrm{C}$ in randomized controlled trial. Patient Educ Couns 2006;64:78-86.

20 Zoffmann V, Prip A, Christiansen AW. Dramatic change in a young woman's perception of her diabetes and remarkable reduction in $\mathrm{HbA1c}$ after an individual course of guided Self-Determination. BMJ Case Rep 2015;2015. doi:10.1136/bcr-2015-209906. [Epub ahead of print: 06 Jul 2015].

21 Zoffmann V, Vistisen D, Due-Christensen M. Flexible guided self-determination intervention for younger adults with poorly controlled Type 1 diabetes, decreased HbA1c and psychosocia distress in women but not in men: a real-life RCT. Diabet Med 2015;32:1239-46.

22 Mathiesen AS, Egerod I, Jensen T, et al. Psychosocial interventions for reducing diabetes distress in vulnerable people with type 2 diabetes mellitus: a systematic review and meta-analysis. Diabetes Metab Syndr Obes 2019;12:19-33.

23 Husted GR, Thorsteinsson B, Esbensen BA, et al. Effect of guided selfdetermination youth intervention integrated into outpatient visits versus treatment as usual on glycemic control and life skills: a randomized clinical trial in adolescents with type 1 diabetes. Trials 2014;15:321.

24 Mohn J, Graue M, Assmus J, et al. The effect of guided selfdetermination on self-management in persons with type 1 diabetes mellitus and $\mathrm{HbA}_{1} \geq 64 \mathrm{mmol} / \mathrm{mol}$ : a group-based randomised controlled trial. BMJ Open 2017;7:e013295.

25 Mathiesen AS, Rothmann MJ, Zoffmann V, et al. Self-determination theory interventions versus usual care in people with diabetes: a protocol for a systematic review with meta-analysis and tria sequential analysis. Syst Rev 2021;10:12

26 Savović J, Jones HE, Altman DG, et al. Influence of reported study design characteristics on intervention effect estimates from randomized, controlled trials. Ann Intern Med 2012;157:429-38.

27 LaFave SE, Granbom M, Cudjoe TKM, et al. Attention control group activities and perceived benefit in a trial of a behavioral intervention for older adults. Res Nurs Health 2019;42:476-82.

28 McCarney R, Warner J, lliffe S, et al. The Hawthorne effect: a randomised, controlled trial. BMC Med Res Methodol 2007;7:30.

29 Polonsky WH, Anderson BJ, Lohrer PA, et al. Assessment of diabetes-related distress. Diabetes Care 1995;18:754-60.

30 Zigmond AS, Snaith RP. The hospital anxiety and depression scale. Acta Psychiatr Scand 1983;67:361-70.

31 Bjelland I, Dahl AA, Haug TT, et al. The validity of the hospital anxiety and depression scale. An updated literature review. J Psychosom Res 2002;52:69-77.

32 Ahroni JH, Boyko EJ. Responsiveness of the SF-36 among veterans with diabetes mellitus. J Diabetes Complications 2000;14:31-9.

33 Bjorner JB, Wallenstein GV, Martin MC, et al. Interpreting score differences in the SF-36 vitality scale: using clinical conditions and functional outcomes to define the minimally important difference. Curr Med Res Opin 2007;23:731-9.

34 Rozental A, Kottorp A, Boettcher J, et al. Negative effects of psychological treatments: an exploratory factor analysis of the negative effects questionnaire for monitoring and reporting adverse and unwanted events. PLoS One 2016;11:e0157503.

35 Rozental A, Kottorp A, Forsström D, et al. The negative effects questionnaire: psychometric properties of an instrument for assessing negative effects in psychological treatments. Behav Cogn Psychother 2019;47:559-72.

36 International Conference on harmonisation of technical requirements for registration of pharmaceuticals for human use, $\mathrm{ICH}$ harmonised guideline: integrated addemdum to ICH E6 (R1): guideline for good clinical practice (ICH-GCP) 2015.

37 Chan A-W, Tetzlaff JM, Altman DG, et al. Spirit 2013 statement: defining standard protocol items for clinical trials. Ann Intern Med 2013;158:200-7.

38 Boutron I, Moher D, Altman DG, et al. Extending the CONSORT statement to randomized trials of nonpharmacologic treatment: explanation and elaboration. Ann Intern Med 2008;148:295-309.

39 Boutron I, Moher D, Altman DG, et al. Methods and processes of the CONSORT group: example of an extension for trials assessing nonpharmacologic treatments. Ann Intern Med 2008;148:W-60-6. 
40 Mathiesen AS. Vulnerable people with type 2 diabetes: implications and feasibility of a guided self-determination intervention for reducing diabetes distress. Department of Public health, Faculty of health and Medical Sciences, University of Copenhagen, 2019.

41 Polonsky WH, Fisher L, Earles J, et al. Assessing psychosocial distress in diabetes: development of the diabetes distress scale. Diabetes Care 2005;28:626-31.

42 Razavi D, Delvaux N, Farvacques C, et al. Screening for adjustment disorders and major depressive disorders in cancer in-patients. $\mathrm{Br} \mathrm{J}$ Psychiatry 1990;156:79-83.

43 Spinhoven P, Ormel J, Sloekers PP, et al. A validation study of the hospital anxiety and depression scale (HADS) in different groups of Dutch subjects. Psychol Med 1997;27:363-70.

44 Ryan RMD, Edward L. Self-determination theory - Basic psychological needs in Motivation, Development and Wellness. The Guilford Press, 2017.

45 Järvinen TLN, Sihvonen R, Bhandari M, et al. Blinded interpretation of study results can feasibly and effectively diminish interpretation bias. J Clin Epidemiol 2014;67:769-72.

46 Gøtzsche PC. Blinding during data analysis and writing of manuscripts. Control Clin Trials 1996;17:285-90.

47 Sperl-Hillen J, Beaton S, Fernandes O, et al. Are benefits from diabetes self-management education sustained? Am J Manag Care 2013;19:104-12.
48 Puhan MA, Frey M, Büchi S, et al. The minimal important difference of the hospital anxiety and depression scale in patients with chronic obstructive pulmonary disease. Health Qual Life Outcomes 2008;6:46.

49 Jakobsen JC, Gluud C, Winkel P, et al. The thresholds for statistical and clinical significance - a five-step procedure for evaluation of intervention effects in randomised clinical trials. BMC Med Res Methodol 2014;14:34

50 Jakobsen JC, Gluud C, Wetterslev J, et al. When and how should multiple imputation be used for handling missing data in randomised clinical trials - a practical guide with flowcharts. BMC Med Res Methodol 2017;17:162.

51 World Medical Association. World Medical association Declaration of Helsinki: ethical principles for medical research involving human subjects. JAMA 2013;310:2191-4.

52 Skoog M SJ, Gluud C, Scheinin M. Transparency and registration in clinical research in the Nordic countries (report), in Nordic trial alliance. Oslo, Norway: NordForsk, 2015: 108.

53 Taichman DB, Sahni P, Pinborg A, et al. Data Sharing Statements for Clinical Trials - A Requirement of the International Committee of Medical Journal Editors. N Engl J Med 2017;376:2277-9.

54 Olesen ML, Duun-Henriksen A-K, Hansson H, et al. A personcentered intervention targeting the psychosocial needs of gynecological cancer survivors: a randomized clinical trial. J Cancer Surviv 2016;10:832-41. 